\title{
An Efficient Approach for Optimization of Nonlinearity Response in Linear Variable Differential Transformer
}

\author{
Dr. Santosh Kumar Sahoo \\ Assoc. Professor, CVR College of Engineering/ EIE Department, Hyderabad, India \\ Email: santosh.kr.sahoo@gmail.com
}

\begin{abstract}
This paper proposes an efficient approach for optimization of non-linearity response reflected in output of Linear Variable Differential Transformer / in Linear Variable Differential Transducer (LVDT). Customarily, the nonlinearity in LVDT was reduced by windings/coils tuning, which was very difficult to get fine tune. This problematic situation can overawe by implementation of the projected scheme. The Functional link artificial neural network (Fun-LANN) has been effectively implemented for nonlinear optimization of the senor's response. The effectiveness of the proposed method is demonstrated through MATLAB simulation and the response has validated with various nonlinear effects. The experimental analysis of the proposed scheme produces high sensitivity, good linearity and insensitivity to excitation variations.
\end{abstract}

Index Terms: LVDT, Fun-LANN, Optimization, Sensor, Tuning, Non-linearity.

\section{INTRODUCTION}

In the field of engineering automation, transducer plays a vital role for measuring, monitoring, recording and control. Various environments are exposed to the transducers for performing the sensing activities. So, the response of the transducer is influenced by the excitation source and operating temperatures. Hence, it is highly necessary for optimizing the issues related to these factors, as a result the sensing element will be smarter enough for reduction of nonlinearity.

Linear Variable Differential Transformer / Linear Variable Differential Transducer (LVDT) is an electrical transformer used for measuring linear displacement. The LVDT converts a position or linear displacement into a proportional electrical signal. This LVDTs are broadly used for displacement, pressure, and flow measurements [1]. LVDT performance is affected by its geometrical structure, Primary and secondary coil arrangement, core material quality, current and frequency variation in excitation source and variation in operating temperature [2]-[3]. Furthermore, the aging factor of transducer is also inviting the nonlinearity. Due to these reasons, the transducers are limited for a particular usable span. The reported nonlinearity is a function of time and depends on several uncertain factors. The linearity is achieved [3] by considering the geometrical structure modification and same also realized by an introduction of twin series connected the secondary coils. Lately, some researchers [4]-[5] have considered the signal conditioning circuit for effective response. The LVDT displays nonlinearity with variation of core null position and at middle position it indicates linearity. By considering this, the proposed scheme is realized with Fun-LANN in order to better linear response.

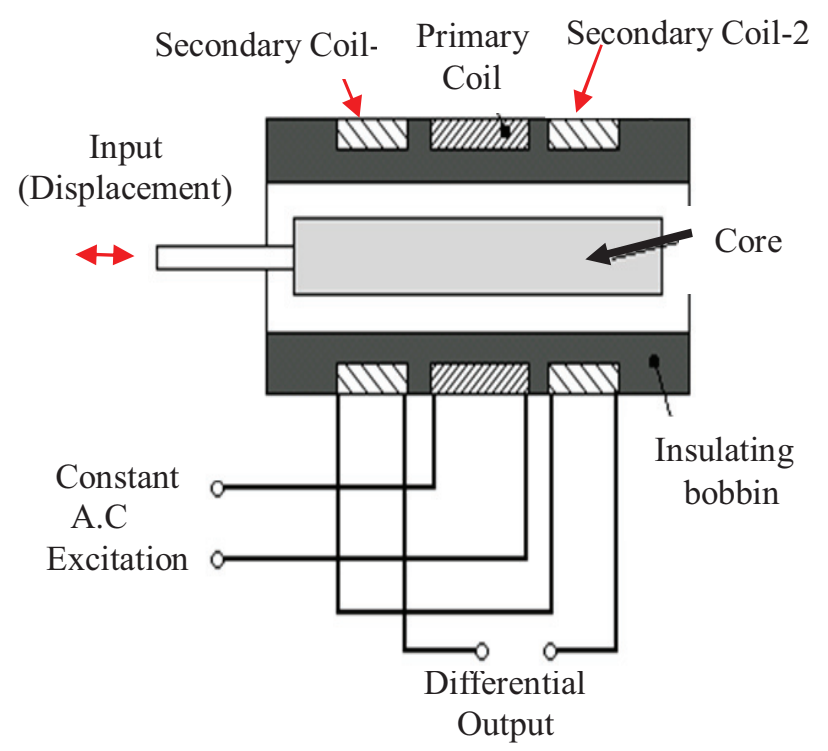

Figure 1. Schematic LVDT arrangement

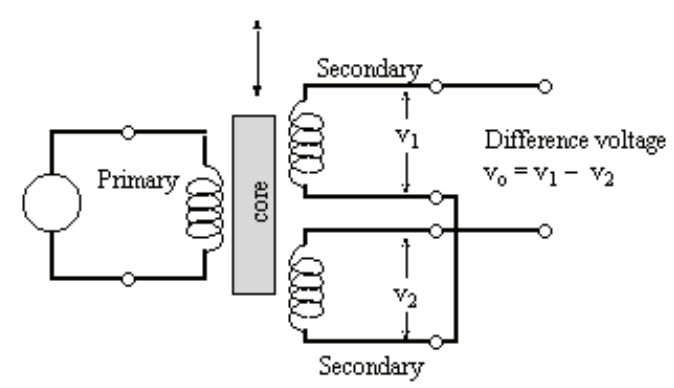

Figure 2. Electrical equivalent circuit arrangement for LVDT

LVDT consists of two coils namely Primary and Secondary. The Primary is excited externally by means of A.C. Excitation with a stable source and two secondary coils are connected in series opposition in order to provide single differential output. Schematic of LVDT arrangement is presented in figure 1. When an A.C. Supply excitation ' $\mathrm{E}_{\mathrm{AC}}$ ' with frequency ' $F_{S}$ ' is applied across primary coil and the core position with respect to primary and two secondary 
windings, the developed output potential ' $\mathrm{V}_{\text {Out }}$ ' is found across the secondary winding.

According to mutual induction principle, the induced potential in one secondary coil is presented in equation-1.

$\mathrm{V}_{\text {Secondary coil }}=V_{S e c}=-N \frac{d \phi}{d r}$

Considering the primary current, the secondary voltage is as per equation-2. $\quad V_{\text {Sec }}=-M \frac{d I_{\text {primary }}}{d t}$

In the above equation, the term ' $\mathrm{N}$ ' represents number of turns in the secondary coil, ' $\emptyset$ ' represents magnetic flux,

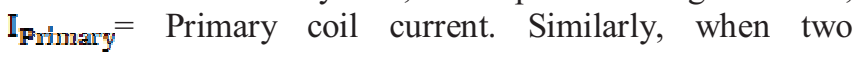
secondary coils are connected in series opposition, the developed output can be presented in equation-3.

$$
V_{\text {OUT-Sec }}=V_{\text {Out-Sec1 }}-V_{\text {Out-Sec } 2}=-\left(M_{1}-M_{2}\right) \frac{d I_{\text {primary }}}{d t}
$$

Where $M_{1}$ and $M_{2}$ are functions of displacement 'x'. Let $M_{1}-M_{2}=M(x)$. At certain range when the function is linear, $M(x)=k x$, as a result the displacement can be presented as per equation $-4 . x=\frac{V_{\text {out }-S_{c}}}{K d I_{\text {Primiary } / d t}}$

The electrical equivalent circuit arrangement for LVDT is presented in Figure 2. In this arrangement, all the loss components are compensated and corresponding output voltage per unit displacement and phase angle are presented in equation -5 and equation- 6 .

$$
\begin{aligned}
& \left|\frac{V_{\text {Out-Secondary }}}{V_{\text {Input }}}\right| \times \frac{1}{x}=\frac{K w R_{m} /\left\{\left(R_{s}+R_{m}\right) R_{p}\right\}}{\sqrt{\left[\left\{1-w^{2}\left(\tau_{m}{ }^{2}+\tau_{p} \tau_{s}\right)\right\}^{2}+w^{2}\left(\tau_{p}+\tau_{s}\right)^{2}\right]}} \\
& \text { And } \phi=90^{0}-\tan ^{-1} \frac{w\left(\tau_{p}+\tau_{s}\right)}{1-w^{2}\left(\tau_{m}{ }^{2}+\tau_{p} \tau_{s}\right)} \\
& \text { Where }_{\tau_{m}}=\frac{M_{1}-M_{2}}{\sqrt{\left(R_{m}+R_{p}\right) R_{p}}} \text { and } \tau_{p}=\frac{L_{p}}{R_{p}} \tau_{s}=\frac{L_{s}}{R_{s}+R_{m}}
\end{aligned}
$$

With input excitation of $V_{\text {input }}$, operating frequency $F_{\text {Input }}$ and meter resistance $R_{m}$. In all LVDT structure, there is some existence of nonlinearity due to some limitations. Hence, the proposed model is developed for overcome these limitations.

\section{Proposed Experimental Setup}

The Figure 3 represents an experimental setup for the proposed structure. In the given experimental setup presented in Figure 3, a stepper motor-controlled displacer is arranged for uniform movement of the LVDT core arm. The controller output tunes the displacement actuator as a result the core in LVDT is moved accordingly and then the nonlinear output of LVDT is again fed to Neural network model (NNM). Meanwhile the actuator output is also acting as setpoint value by the NNM. With the input and setpoint values, the NNM weights are revised in order to optimize an error. This NNM also enhances the linear response of the LVDT in a significant way. Researchers have found a useful alternative for artificial neural network as functional link artificial neural network (Fun-LANN) [6-7]. This FunLANN has advantages over ANN like it takes less computational time and a simple hardware structure.

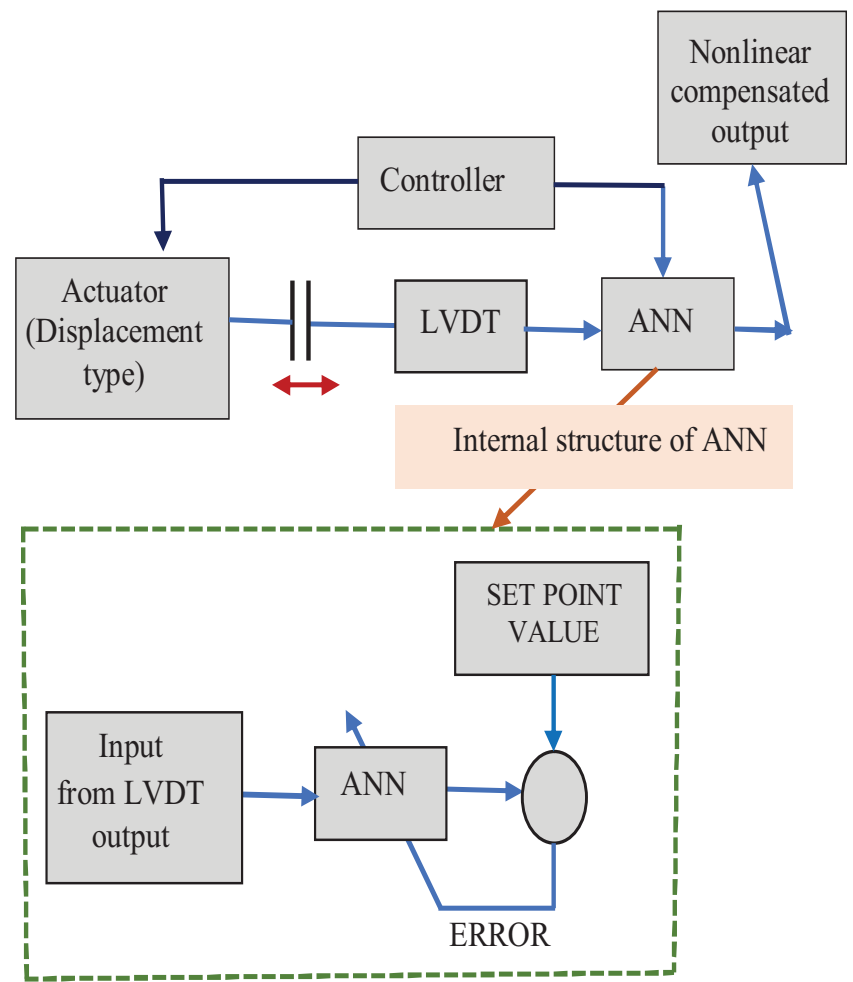

Figure 3. An experimental setup for the proposed structure

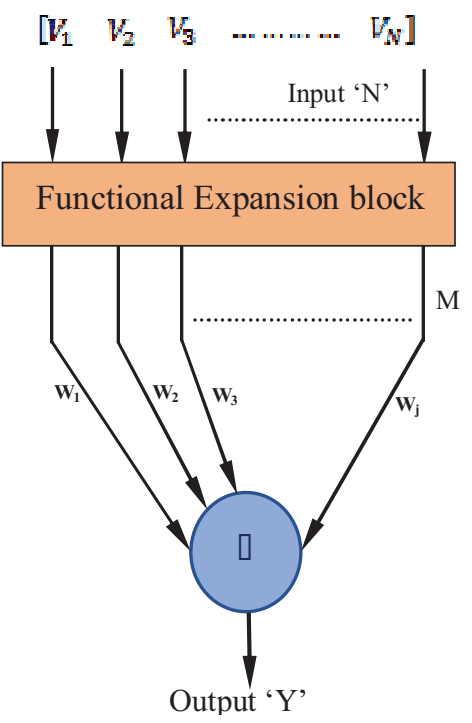

Figure 4. Simplified Fun-LANN structure

A simplified Fun-LANN structure is presented in Figure 4, where ' $\mathrm{N}$ ' inputs are fed to the functional expansion block to generate $M$ functionally expanded signals and these signals are combined with $\mathrm{M}$-element weight vector to produce a single output.

In this article, a trigonometric functional expansion is preferred for Fun-LANN. If all the polynomials of $\mathrm{x}^{\text {th }}$ order with respect to an orthogonal system, the best approximation in the metric space is presented by the partial summation of Fourier series of $\mathrm{x}^{\text {th }}$ polynomial with respect to system. 
Hence, the trigonometric polynomial basis function is expressed as per equation (7).

$$
M=\left\{V_{0}, \operatorname{Sin}(\pi V), \operatorname{Cos}(\pi V), \operatorname{Sin}(2 \pi V), \operatorname{Cos}(2 \pi V) \ldots \ldots \operatorname{Sin}(x \pi V), \operatorname{Cos}(x \pi V)\right\}
$$

The equation 7 signifies the compact representation of the function in mean square sense.

Let the LVDT output is extended functionally ' $V_{\text {out }}$ '. Now functionally extended ' $M$ ' vector is linearly joint with weight matrix by subsequent ways [8-9]

$$
Y=\sum_{j=1}^{M} M_{f} w_{j}+1 x
$$

Where $\mathrm{o}=$ bias term and

$$
M_{j}=\left\{\begin{array}{lc}
V_{c} & j=1 \\
\sin \left(l \pi V_{0}\right), & j>1, j \text { gven } \\
\cos \left(2 \pi V_{a}\right), & j>1, j \text { odd }
\end{array}\right.
$$

Where $1 \leq l \leq x, x$ is the order of expansion. Now the error signal can be defined as

$$
\mathrm{E}(\mathrm{n})=\mathrm{D}(\mathrm{n})-\sum_{\mathrm{N}}=1 \mathrm{M}_{\mathrm{i}} \mathrm{w}_{\mathrm{i}}
$$

Where $D(n)$ is set point value or desired signal or equivalent control signal applied to displacement actuator and ' $\mathrm{n}$ ' is time index. Let the cost function ' $\zeta$ ' as residual noise power and it is presented as $[10] \zeta=\mathrm{E}\left[E^{2}(n)\right]$

The weight vector $w_{j}$ may be adjusted as the per following in order to optimization of mean square error (MSE) $\zeta$

$w_{j+1}=w_{j}-\frac{\mu}{2} \nabla(\mathrm{n})$

Where $\nabla(n)$ is an instantaneous estimation of gradient of $\zeta$ with respect to weight vector $w_{j}$.

Hence, $w_{j+1}=w_{j}+\mu E(n) M(n)$

Where ' $\mu$ ' denotes the step size and it controls the convergence speed of the algorithm.

\section{EXPERIMENTAL SimUlation}

Using experimental data sets, the response of the proposed model has been studied and this experimental data are collected from an LVDT with and without combination of the proposed model. The LVDT structure considered here having 3299 turns in the secondary coils, wound uniformly on each side isolated by Teflon ring. The core dia used in the proposed model is about $4.39 \mathrm{~mm}$ and length of core is about $4.6 \mathrm{~mm}$. The primary coil excited by an excitation source of $10.5 \mathrm{~V}_{\text {P-P }}$ with operating frequency of $5.2 \mathrm{KHz}$. Resistance of Primary winding is $267 \Omega$ and resistance of two Secondary coil are $423.5 \Omega$ and $412.8 \Omega$. The two secondary coils are wound in opposite directions (Series Opposition) for single differential output. The experimental measured values are presented in Table-1. The above data are normalized and utilized by Fun-LANN. The normalized LVDT output be considered as input to Fun-LANN and Fun-LANN output is equated with the normalized displacement as per Table-1data. Two experimental analysis are implemented with the same data set. The $1^{\text {st }}$ test has performed through low functional expansion value (i.e $x=9$ ) and in $2^{\text {nd }}$ testing through high value of functional expansion (i.e $x=109$ ). Figure-5 represents response of LVDT and the non-linear compensator for $1^{\text {st }}$ test. Similarly, the figure- 6 shows the same for high value of functional expansion (i.e $x=109$ ).
From figure 4, it is concluded that there is substantial upgrading of linearity thru Fun-LANN founded compensator

\begin{tabular}{|c|c|c|}
\hline $\begin{array}{l}\text { Sl. } \\
\text { No }\end{array}$ & Displacement in $\mathrm{mm}$ & $\begin{array}{l}\text { LVDT Output Voltage }\left(V_{o}\right) \\
\text { in } \mathrm{mV}\end{array}$ \\
\hline 1 & -26 & 3.985 \\
\hline 2 & -24 & 3.852 \\
\hline 3 & -22 & 3.732 \\
\hline 4 & -20 & 3.723 \\
\hline 5 & -18 & 3.685 \\
\hline 6 & -16 & 3.584 \\
\hline 7 & -14 & 3.452 \\
\hline 8 & -12 & 3.212 \\
\hline 9 & -10 & 2.985 \\
\hline 10 & -8 & 2.125 \\
\hline 11 & -4 & 1.118 \\
\hline 12 & -2 & 0.952 \\
\hline 13 & -1 & 0.829 \\
\hline 14 & Null Position & 0.185 \\
\hline 15 & 1 & 0.812 \\
\hline 16 & 2 & 0.978 \\
\hline 17 & 4 & 2.975 \\
\hline 18 & 8 & 2.145 \\
\hline 19 & 10 & 2.998 \\
\hline 20 & 12 & 3.412 \\
\hline 21 & 14 & 3.652 \\
\hline 22 & 16 & 3.784 \\
\hline 23 & 18 & 3.885 \\
\hline 24 & 20 & 3.926 \\
\hline 25 & 22 & 3.981 \\
\hline 26 & 24 & 3.982 \\
\hline 27 & 26 & 3.985 \\
\hline
\end{tabular}
through a greater numeral of functional expansions.

TABLE I.

EXPERIMENTAL MEASURED VALUES

\begin{tabular}{|c|c|c|c|}
\hline $\begin{array}{l}\text { S1. } \\
\text { No }\end{array}$ & $\begin{array}{l}\text { Displacement } \\
\text { in } \mathrm{mm}\end{array}$ & $\begin{array}{l}\text { LVDT Output } \\
\text { Voltage }\left(V_{o}\right)\end{array}$ & $\begin{array}{l}\text { Output Voltage of } \\
\text { proposed Model } \\
\text { (LVDT with Fun- } \\
\text { LANN) in mV }\end{array}$ \\
\hline 1 & -26 & 3.985 & 4.985 \\
\hline 2 & -24 & 3.852 & 4.852 \\
\hline 3 & -22 & 3.732 & 4.732 \\
\hline 4 & -20 & 3.723 & 3.883 \\
\hline 5 & -18 & 3.685 & 3.868 \\
\hline 6 & -16 & 3.584 & 3.758 \\
\hline 7 & -14 & 3.452 & 3.652 \\
\hline 8 & -12 & 3.212 & 3.512 \\
\hline 9 & -10 & 2.985 & 3.525 \\
\hline 10 & -8 & 2.125 & 3.125 \\
\hline 11 & -4 & 1.118 & 2.118 \\
\hline 12 & -2 & 0.952 & 1.952 \\
\hline 13 & -1 & 0.829 & 1.829 \\
\hline 14 & Null Position & 0.185 & 0.175 \\
\hline 15 & 1 & 0.812 & 1.812 \\
\hline 16 & 2 & 0.978 & 1.978 \\
\hline 17 & 4 & 2.975 & 3.975 \\
\hline 18 & 8 & 2.145 & 3.984 \\
\hline 19 & 10 & 2.998 & 3.998 \\
\hline 20 & 12 & 3.412 & 4.412 \\
\hline 21 & 14 & 3.652 & 4.652 \\
\hline 22 & 16 & 3.784 & 4.784 \\
\hline 23 & 18 & 3.885 & 4.885 \\
\hline 24 & 20 & 3.926 & 4.926 \\
\hline 25 & 22 & 3.981 & 4.981 \\
\hline 26 & 24 & 3.982 & 4.982 \\
\hline 27 & 26 & 3.985 & 4.985 \\
\hline
\end{tabular}

TABLE II.

COMPARISON OF EXPERIMENTAL MEASURED VALUES 
The proposed model (FANN) with 109 functional expansion is achieving better linearity.

Table II summarizes the comparison statement between the output response of LVDT with and without considering Fun-LANN (Proposed) model.

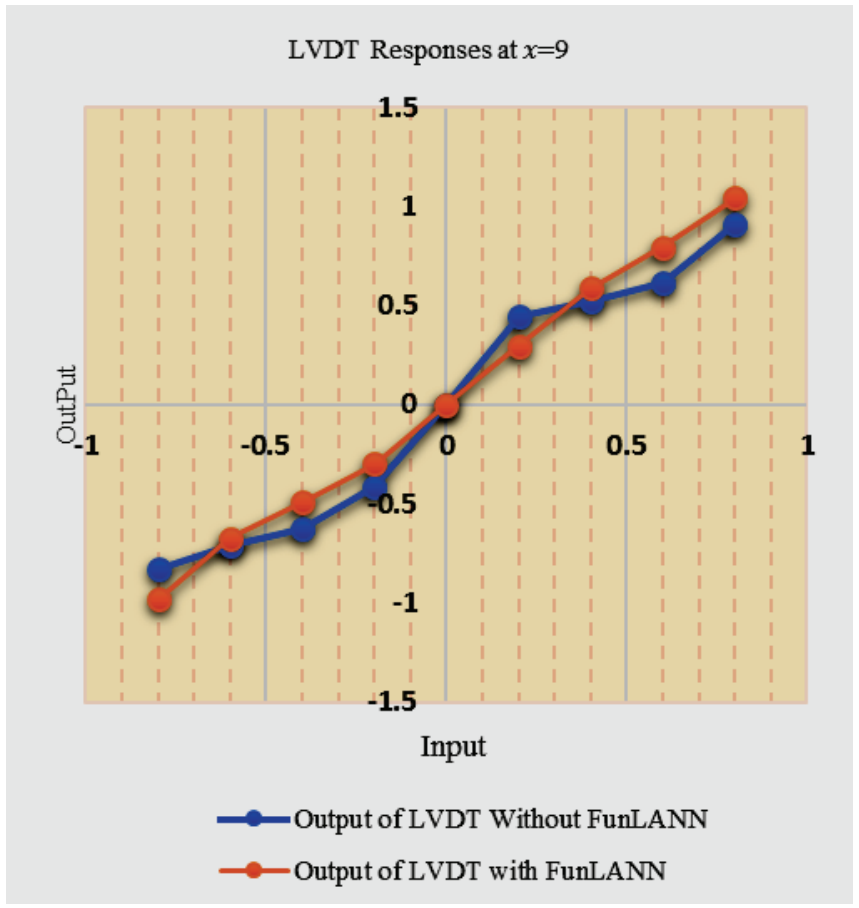

Figure 5. Response of LVDT at $x=9$

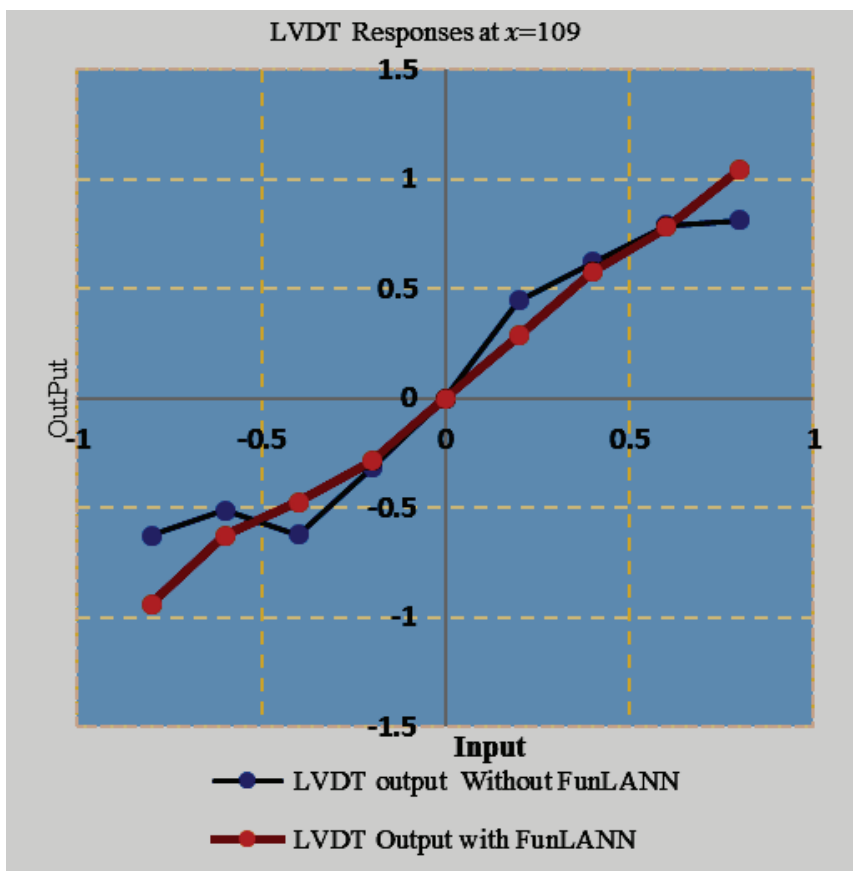

Figure 6. Response of LVDT at $x=109$

The response of the LVDT with and without considering the proposed scheme at various functional expansion values (i.e $x=9$ and $x=109$ ) are detailed in Table III and Table IV respectively. From these data, it is resolved that the proposed model has significant response in terms of linearity corresponding to input displacement variations.

TABLE III.

COMPARISON OF EXPERIMENTAL MEASURED VALUES AT $x=9$

\begin{tabular}{|c|c|c|}
\hline $\begin{array}{c}\text { Input } \\
\text { Displacement }\end{array}$ & $\begin{array}{c}\text { Output of LVDT } \\
\text { Without Fun-ANN }\end{array}$ & $\begin{array}{c}\text { Output of LVDT with } \\
\text { Fun-LANN }\end{array}$ \\
\hline-0.8 & -0.825 & -0.981 \\
\hline-0.6 & -0.712 & -0.675 \\
\hline-0.4 & -0.621 & -0.494 \\
\hline-0.2 & -0.418 & -0.297 \\
\hline 0 & 0 & 0 \\
\hline 0.2 & 0.445 & 0.298 \\
\hline 0.4 & 0.521 & 0.589 \\
\hline 0.6 & 0.612 & 0.795 \\
\hline 0.8 & 0.912 & 1.045 \\
\hline
\end{tabular}

TABLE IV

COMPARISON OF EXPERIMENTAL MEASUREd VALUES AT $x=109$

\begin{tabular}{|c|c|c|}
\hline Input Displacement & $\begin{array}{c}\text { LVDT output (mV) } \\
\text { Without Fun-ANN }\end{array}$ & $\begin{array}{c}\text { LVDT Output } \\
(\mathrm{mV}) \text { with Fun- } \\
\text { LANN }\end{array}$ \\
\hline-0.8 & -0.625 & -0.941 \\
\hline-0.6 & -0.512 & -0.625 \\
\hline-0.4 & -0.621 & -0.474 \\
\hline-0.2 & -0.318 & -0.287 \\
\hline 0 & 0 & 0 \\
\hline 0.2 & 0.445 & 0.288 \\
\hline 0.4 & 0.621 & 0.579 \\
\hline 0.6 & 0.792 & 0.783 \\
\hline 0.8 & 0.812 & 1.045 \\
\hline
\end{tabular}

\section{CONCLUSIONS}

In this article, a simple and an effective way of designing the high linearity LVDT based displacement sensing structure is proposed. The proposed Fun-LANN is successfully applied for nonlinear optimization of the LVDT. The Detailed implementation procedure for training the Fun-LANN model is outlined properly. The proposed scheme also makes the sensing element smarter enough for auto-correction of nonlinear response. The proposed scheme is also suitable for nonlinear compensation of other sensing element.

\section{REFERENCES}

[1] C. D. Johnson and H. Al. Richeh, Highly accurate resistance deviation to frequency converter with programmable sensitivity and resolution, IEEE transactions on Instrumentation and Measurement, Vol.IM-35, pp.178181,1986

[2] D. Patranabis, S. Ghosh and C. Bakshi, Linearizing transducers characteristics, IEEE Transactions on Instrumentation and Measurement, Vol.37, pp.66-69,1988 
[3] G. Bucci and C. Landi, Metrological characterization of a contactless smart thrust and speed sensor for linear induction motor testing, IEEE Transactions on Instrumentation and Measurement, Vol.45, pp.493-498, 1996

[4] Giovanni Bucci, Marco Faccio and Carmine Landi, New ADC with piecewise Linear Characteristics: Case StudyImplementation of a smart Humidity sensor, IEEE Transactions on Instrumentation and Measurement, Vol.49 (6), 2000

[5] J. M. Dias Pereira, O. Postolache and P. M. B. S. Girao, A Digitally programmable A/D Converter for smart sensor Applications, IEEE Transactions on Instrumentation and Measurement, Vol56(1),2007.

[6] Wu. Shang-The, Mo. Szu-Chieh and Wu. Bo-Siou, An LVDT based self-actuating displacement transducer, Sensors and actuators, Vol.142, PP.558-564,2008
[7] Hermann K P Neubert, "Instrument Transducers: An introduction to their performance and design", $2^{\text {nd }}$ edition, Oxford University press.

[8] S. Haykin, "Neuraal Networks: A Comprehensive Foundation", Pearson Education Asia,2002.

[9] J. M. D. Pereira, O. Postolache and P. M. B. S. Giirao, "A digitally programmable A/D Converter for smart Sensors Applications, IEEE Transactions on Instrumentation and Measurement", Vol. 56, No.1, 2007

[10] Giovanni Bucci, Marco Faccio, and Carmine Landi, "New ADC with piecewise Linear characteristic: Case StudyImplementation of a smart humidity sensor", IEEE transactions on Instrumentation and Measurement, Vol.49, No.6, 2000. 